\title{
ROBUST CONTROL DESIGN OF AN ACTIVATED SLUDGE PROCESS
}

\author{
P. G. GEORGIEVA ${ }^{1 *}$ AND S. FEYO DE AZEVEDO ${ }^{2}$ \\ ${ }^{1}$ Institute of Control and System Research, Bulgarian Academy of Sciences, P. O. Box 79, 1113 Sofia, Bulgaria \\ ${ }^{2}$ Departmento de Engengharia Química, Faculdade de Engenharia, Universidade do Porto, R. dos Bragas, 4099 Porto Codex, \\ Portugal
}

\section{SUMMARY}

A $H$-inf control strategy is presented for a robustly performing activated sludge process. In operational terms, the objective is to conduct the process imposing that the biomass concentration in the recycle stream follows closely a time-varying, process-dependent, reference signal. The corresponding control objective is described as the development of a robust reference-tracking control structure with the best possible disturbance compensation, able to perform with noisy measurements and able to cope with variations in key process model parameters. The proposed algorithm embeds an estimation of states by solving the Riccati equation and avoids parameter estimation by assuming their variability within known bounds. Results are presented which show a favourable behaviour of the robust controller in comparison with a conventional PI control structure. Copyright (C) 1999 John Wiley \& Sons, Ltd.

Key words: $H$-inf robust control; activated-sludge process; model uncertainty

\section{INTRODUCTION}

Wastewater engineering represents at present time a subject area of worldwide interest, for reasons of the public health, economic and social issues to which it is closely associated. The main subjects within wastewater engineering, namely the core topics 'treatment, disposal and reuse of wastewater' are extensively treated in major textbooks. ${ }^{1,2}$ Many other relevant scientific and technical contributions in this domain are a direct consequence of the effort of several task groups, such as those under the auspices of the International Association on Water Pollution Research and Control (IAWPRC). ${ }^{3-5}$

This paper was submitted for publication by editor M. J. Grimble

\footnotetext{
* Correspondence to: P. G. Georgieva, Institute of Control and System Research, Bulgarian Academy of Sciences, P. O. Box 79, 1113 Sofia, Bulgaria. E-mail: pgeorgieva@iusi.bas.bg

Contract/grant sponsor: NATO Science Fellowship

Contract/grant number: 2540

Contract/grant sponsor: National Foundation "Scientific Investigations"

Contract/grant number: TN-715/97
}

CCC 1049-8923/99/130949-19\$17.50

Received 1 February 1999

Copyright (C) 1999 John Wiley \& Sons, Ltd.

Revised 28 February 1999

Accepted 29 June 1999 
A wastewater flow may be made of essentially four types of flows, viz: (i) domestic (sanitary) wastewater; (ii) industrial wastewater; (iii) infiltration water and (iv) storm water. The general question to address is that which contaminants must be removed (and to what extent they must be removed) in order to protect the environment. The composition of industrial wastes depends strongly on the specific sources. Wastes may contain suspended solids, biodegradable organic compounds, refractory organic (surfactants and pesticides), pathogens, priority pollutants (e.g. carcinogenic or highly toxic compounds), nutrients (nitrogen and phosphates, pollutants when in excess), dissolved inorganic (e.g. calcium or sodium in excess) and heavy metals. Consequently, the question, which contaminants to remove, has specific problem-dependent answers.

Wastewater treatment involves mainly three groups of operations or processes - (i) physical unit operations, such as sedimentation and filtration; (ii) chemical processes, of which chemical precipitation and adsorption are examples; and (iii) biological processes, where removal is achieved through some form of biological activity. Most often a combination of the three types will have to be performed in a plant.

The main application of biological treatment processes is on the removal of carbonaceous organic matter. Biological treatments are also employed for the coagulation and removal of non-settleable colloidal solids and for the control of nutrient concentration (namely nitrogen through nitrification/dentrification processes and phosphorus) which should be kept within acceptable low levels.

Biological treatment processes are classified as aerobic or anaerobic, depending on the presence or absence of oxygen. The individual processes can be further classified as of the suspended-growth type, if the organic matter and other constituents are kept in suspension within the liquid, or of the attached-growth type in which case the relevant micro-organisms are kept attached to some insert medium.

\subsection{The activated sludge process}

Aerobic suspended-growth treatment processes can be classified in four groups-(i) the activated-sludge process; (ii) aerated lagoons; (iii) sequencing batch reactors; and (iv) aerobic digesters.

Wastewater processing by means of activated sludge is the most widespread biological treatment practice, particularly for wastewater flows where domestic wastes predominate. It involves the production of an activated mass of microorganisms, which, aerobically, will stabilize the waste.

There are today many versions of the original process (proposed in England, back in $1914,{ }^{6}$ but fundamentally they are similar. In a typical unit organic wastes are fed to a bioreactor (Figure 1) where they are removed through aerobic digestion, a biochemical oxidation process which involves several biological reactions occurring simultaneously. ${ }^{1}$

The bioreactor effluent is then fed to a settler for sedimentation of the organic solid material. The upper output flow from the settler $F_{\mathrm{e}}$ is the purified water, free of solids. The lower output flow is divided into a recycle stream and a surplus biomass removed from the process. The recycle stream is fed back to the bioreactor to increase the concentration of biomass therein and to stimulate further oxidation of organic solids.

A first relevant design aspect with implication in process operation concerns the degree of mixing effectively achieved in the aeration basin. Expectedly it ranges from completely mixed to plug flow regimes. ${ }^{7}$ Most often the design is such that the actual regime is near to the completely 


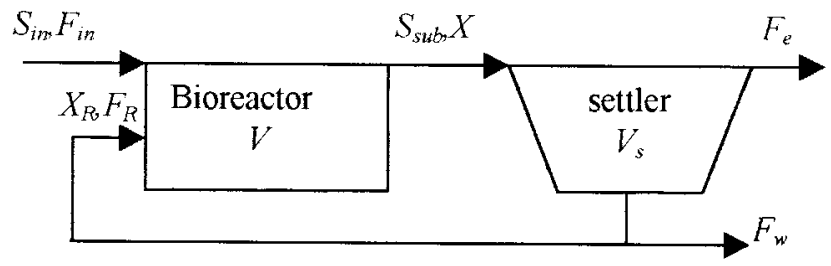

Figure 1. Activated sludge process with settler

mixed reactor model. In many respects the aeration basin is comparable to a conventional fermentation reactor or chemostat. However, it should be noted that the purpose of the process is not to produce microbial biomass or a particular metabolite but to decompose incoming waste materials. In fact and since biomass is to be removed and treated in a subsequent stage, its production should be minimized and also controlled in order to enhance the settling characteristics of the biological floc.

\subsection{Process operation}

There are alternative strategies for the operation of this type of industrial units. ${ }^{8,9}$

Dissolved oxygen and/or substrate concentration in the recycle stream are potential measurement variables. The organic contents may be estimated by independent and to some extent complementary tests ${ }^{2}$ of the Biological Oxygen Demand (BOD), the Chemical Oxygen Demand (COD) and the Total Organic Carbon (TOC). The relationship among these measures depends on the nature of the wastewater. The BOD is the amount of oxygen $(\mathrm{mg} / \mathrm{lor} \mathrm{mg} / \mathrm{kg}) \mathrm{used}$ by microorganisms in the biochemical oxidation of organic matter; the BOD takes a long time to be obtained (the most widely used parameter is the 5-day BOD). The COD is the amount of oxygen $(\mathrm{mg} / \mathrm{l}$ or $\mathrm{mg} / \mathrm{kg}$ ) required to oxidize, by chemical means, organic carbon compounds completely to $\mathrm{CO}_{2}$ and $\mathrm{H}_{2} \mathrm{O}$. It can be obtained in about $3 \mathrm{~h}$. For this reason, for each type of waste the correlation between BOD and COD is independently studied and the COD is the measure routinely made.

The recycle flow rate and/or the aeration rate represents possible manipulated variables.

In the relevant literature ${ }^{10,11}$ several different control problems have been considered, covering all aspects from the dissolved oxygen and the specific utilisation rate control to more comprehensive strategies involving sludge recycle and sedimentation control.

An efficient way to optimize the settling velocity of the sludge and therefore the removal of organic matter in the water is to control the concentration of the biomass in the recycle flow. It should be maintained close to a time-varying reference signal proportional to the measured influent flow rate. ${ }^{12}$ This operating policy is implemented in the present work: the recycle biomass concentration is to track a time-varying reference signal, by actuating on the recycle flow rate.

\subsection{Control problem and strategy}

This reference-tracking problem should take into account and cope with the presence of disturbances such as the inlet flow rate (measurable) and the concentration of wastes in the inlet streams (unmeasurable). Measurement noise represents an unavoidable difficulty in this type of industrial environment and with the measurement technology available and employed. The control task is further hampered by the nonlinearity of the process dynamics and by the known 
kinetic variability which characterizes biological systems. The design problem is thus quite challenging.

Several control strategies are reported to have been applied to wastewater process operation: ${ }^{8,13}$ these vary from the classical PI and PID control algorithms, through the advanced linear quadratic optimal control, to the more sophisticated self-tuning control, adaptive linearizing control ${ }^{9,14}$ and knowledge-based (or expert) systems. ${ }^{13}$

Though many significant applications of advanced robust control are reported in the area of chemical processes ${ }^{15,16}$ very few results are available for biological systems. The $H$-inf approach seems to be very promising since it fits in the type of requirements and difficulties posed by this class of systems. This paper reports on the application of the $H$-inf approach to the activated-sludge process. The design is based on a linearized process model, which is augmented with a norm bounded uncertainty description to capture the discrepancy between the simplified model and the process and the variation in the process parameters. All performance specifications are defined in the frequency domain. The design aim is to find the best possible controller structure under input constraints, which optimize between the conflicting objectives (tracking and robustness), assuming the worst case of disturbance action.

The design procedure to be presented goes through the following steps:

- Setting-up and linearization of a nonlinear process model;

- Description of performance specifications using weighing function;

- Two port state-space representation of the augmented process model;

- State-space $H$-inf control design;

- Robust analysis in the frequency domain (frequency-domain characteristics);

- Computer simulation of the controlled nonlinear process (time-domain characteristics).

\section{PROCESS MODELLING}

In an activated sludge unit a community of microorganisms is supplied with organic matter and oxygen. The mixture of sludge and water in the aeration basin (Figure 1) is called mixed-liquor (ML). The biological phenomena concerning the degradation of the carbonaceous organic matter can be classified in two steps of oxidation and synthesis: (i) the microorganisms consumes organic matter and under proper environmental conditions transform the biodegradable pollutant, by means of aerobic metabolism, into carbon dioxide, water and minerals, generating energy; and (ii) this energy is used for the synthesis of the remainder of the organic waste into new cells.

The removal of nitrogen is performed by a nitrification/denitrification process. The nitrification step, where ammonia is oxidized to nitrites and further to nitrates, often takes place in the aeration basin of the activated-sludge unit. In less frequent situations, nitrification is carried out in a separated reactor following the conventional activated sludge treatment.

Several mathematical models are already available ${ }^{17,18}$ which take into account the full structure of the microbial dynamics and give a detailed picture of the phases in the conventional process: (i) biodegradation of organic matter; (ii) nitrification (if applicable); (iii) dissolved oxygen utilization and (iv) sludge sedimentation.

The first aspect of modelling is the microbial kinetics model, which represents the interaction between the carbonaceous substrate and the sludge biomass. The sedimentation dynamics is not directly concerned with the biochemical reactions, but rather considers the double function of the 


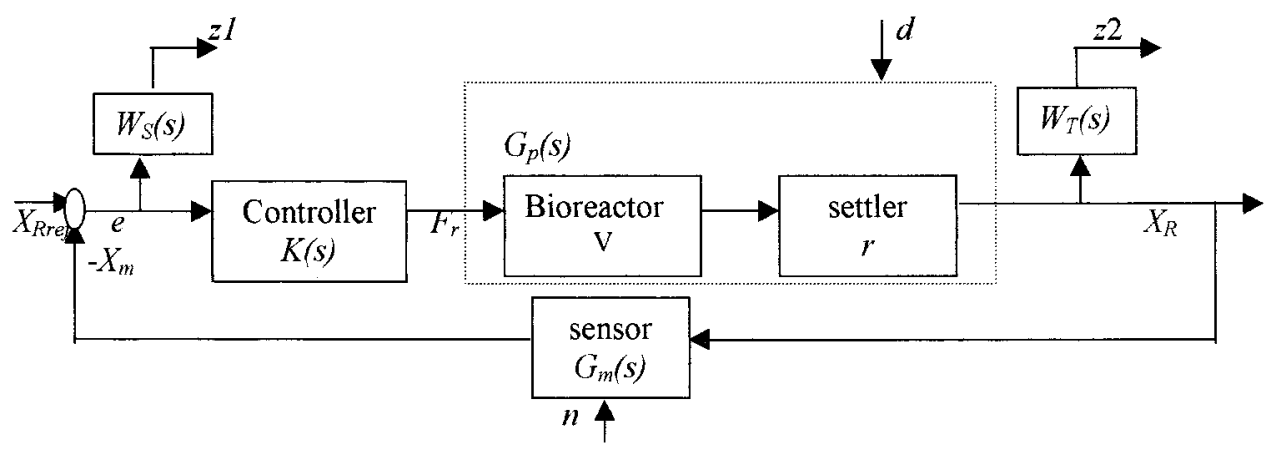

Figure 2. Feedback control structure

settler, separating the bioflocs from the liquid in order to produce a solid-free effluent and thickening the biomass at the bottom of the settler to be recycled back into the aerator.

A detailed description of all reactions arising in the bioreactor would lead to a high-order model of differential equations. ${ }^{19}$ For the control strategy developed in this work a simplified reduced order model is sufficient, as far as it preserves the structural properties of the process:

- Considering the availability of the appropriate kinetic model for waste degradation and assuming that the oxygen availability and transfer is not a limiting step, only the first (i) and the last (iv) process phases are explicitly considered at the modelling stage.

- Taking into account the objectives underlying the control design it is not relevant to consider separately all different biomass and substrate concentrations in the recycle bioreactor. Consequently, the activated-sludge process can be treated as a single-substrate, singlebiomass system, the former being given by Chemical Oxygen Demand measurement.

- A complete continuous-flow model can be obtained combining both the kinetics of the main process streams and the appropriate input-output transport terms. Hereafter, the bioreactor is considered to be perfectly mixed so that the concentration of each component is spatially homogeneous.

- An usual stage of simplification involves accepting Quasi-Steady-State Assumptions (QSSA) applied to substrates and products, which are characterized by fast dynamics. ${ }^{20,21}$

The overall set of equations describing the system represented in Figure 2 is established as follows:

Mass balance to the bioreactor

$$
\begin{aligned}
\dot{X}(t) & =\left(\mu\left(S_{\mathrm{sub}}\right)-\frac{F_{\mathrm{in}}(t)+F_{\mathrm{R}}(t)}{V}\right) X(t)+\frac{F_{\mathrm{R}}(t)}{V} X_{\mathrm{R}}(t) \\
\dot{S}_{\mathrm{sub}}(t) & =-\frac{1}{Y} \mu\left(S_{\mathrm{sub}}\right) X(t)+\frac{F_{\text {in }}(t)}{V} S_{\mathrm{in}}-\frac{F_{\text {in }}(t)+F_{\mathrm{R}}(t)}{V} S_{\text {sub }}(t)
\end{aligned}
$$

where $X$ is the active microbial biomass, which is considered as the total amount of the sludge present in the mixed liquor and is represented by the Mixed Liquor Suspended Solids (MLSS), $S_{\text {sub }}$ is the substrate measured by the Chemical Oxygen Demand (COD), $V$ is the bioreactor 
volume, $F_{\mathrm{R}}$ represents the recycle flow rate (manipulated variable), $F_{\mathrm{in}}$ is the wastewater feed rate and $S_{\text {in }}$ is the inlet substrate concentration (potential disturbance, also expressed as COD). $\mu($.$) is$ the specific growth rate, which is the key parameter for description of biomass growth and substrate consumption of the reaction. It is modelled by a Monod-type equation

$$
\mu\left(S_{\mathrm{sub}}(t)\right)=\frac{\mu_{\mathrm{m}} S_{\mathrm{sub}}(t)}{K_{\mathrm{m}}+S_{\mathrm{sub}}(t)}
$$

where $\mu_{\mathrm{m}}$ is the maximum growth rate and $K_{\mathrm{m}}$ is the half-saturation constant of biodegradable organic matter. It is the concentration of the substrate at which $\mu=\mu_{\mathrm{m}} / 2$.

Mass balance to the settler

It is supposed that the settler is such that the whole biomass will settle, none leaving the unit in the upper output stream. The concentration of the biomass in the recycle stream depends on the specific settler used. The dynamics of the settler can be described by the following mass balance equation: ${ }^{9}$

$$
\dot{X}_{\mathrm{R}}(t)=\left(\frac{F_{\text {in }}(t)+F_{\mathrm{R}}(t)}{V_{\mathrm{S}}}\right) X(t)-\left(\frac{F_{\mathrm{W}}(t)+F_{\mathrm{R}}(t)}{V_{\mathrm{S}}}\right) X_{\mathrm{R}}(t)
$$

where $F_{\mathrm{W}}$ is the waste flow rate and $V_{\mathrm{S}}$ is the settler volume.

The settler has a first-order dynamics, which is much faster, than the bioreactor dynamics and can be consequently neglected. Moreover, assuming that a constant ratio of output to input solids concentration is maintained, the description of the settler behaviour can be approximated ${ }^{12}$ by

$$
X_{\mathbf{R}}(t)=r(t) X(t)
$$

where the parameter $r$ is considered as varying, with known bounds to take into account the error of the simplification accepted.

The manipulated variable is the recycle flow rate. Substituting equation (4) into equation (1) leads to a nonlinear relationship between the process-controlled variable and the manipulated variable,

$$
\dot{X}_{\mathbf{R}}(t)=\left(\mu\left(S_{\mathrm{sub}}\right)-\frac{F_{\text {in }}(t)}{V}\right) X_{\mathbf{R}}(t)+\frac{X_{\mathbf{R}}(t)}{V}(r(t)-1) F_{\mathbf{R}}(t)
$$

Process measurements

It is assumed that the measurements are available for the biomass concentration in the recycle stream, either inferred from measurements of the concentration of $\mathrm{CO}_{2}$ and $\mathrm{O}_{2}$ gases in the bioreactor, ${ }^{22}$ or taken from on-line turbimetric measurements. ${ }^{19}$ The dynamics of this non-direct measurement of the recycle biomass concentration is expressed by the first-order transfer function,

$$
X_{\mathrm{m}}(s)=\frac{k_{\mathrm{m}}}{T_{\mathrm{m}} s+1} X_{\mathrm{R}}(s)+n(s)
$$

where $n(s)$ represents a random high-frequency measurement noise introduced in the system.

The time-varying control reference

A basic assumption is that the plant input has a diurnal periodicity. This approach is well suited for plants dealing with domestic wastes whose inputs are known to be consistently 
periodic. ${ }^{23}$ Therefore, the reference concentration of the biomass in the recycle flow is to be maintained close to a time-varying reference signal which is proportional to the measured influent flow rate,

$$
X_{\mathrm{Rref}}(t)=k_{\mathrm{ref}} F_{\mathrm{in}}(t)
$$

\section{MODEL LINEARIZATION}

Applying the robust control strategy, the process dynamics (equation (5)) is presented as a linear model with uncertain parameters, ${ }^{12}$

$$
X_{\mathbf{R}}(t)=a X_{\mathbf{R}}(t)+b F_{\mathbf{R}}(t)
$$

where

$$
a=\left(\mu-\frac{F_{\mathrm{in}}}{V}\right), \quad b=\frac{X_{\mathrm{R}}}{V}(r-1)
$$

With the exception of the volume all other parameters in $a$ and $b$ are accepted to vary between known bounds. Consequently, it follows that,

$$
a \in\left[a_{\min }, a_{\max }\right], \quad b \in\left[b_{\min }, b_{\max }\right]
$$

Applying Laplace transforms to equation (8), with zero initial conditions, leads to

$$
X_{\mathrm{R}}(s)=\frac{b}{s-a} F_{\mathrm{R}}(s)=G_{\mathrm{p}}(s) F_{\mathrm{R}}(s)
$$

where

$$
G_{\mathrm{p}}(s)=\frac{b}{s-a}
$$

is the transfer function of the process. The uncertain parameters can be expressed as

$$
\begin{gathered}
G_{\mathrm{p}}(s)=\frac{b_{\mathrm{nom}}+\Delta b}{s-\left(a_{\mathrm{nom}}+\Delta a\right)} \\
b_{\mathrm{nom}}=\frac{b_{\text {max }}+b_{\min }}{2}, \quad \Delta b=\gamma_{b} \Delta_{b}, \quad \gamma_{b}=\frac{b_{\text {max }}-b_{\min }}{2}, \quad\left|\Delta_{b}\right|<1 \\
a_{\mathrm{nom}}=\frac{a_{\mathrm{max}}+a_{\min }}{2}, \quad \Delta a=\gamma_{a} \Delta_{a}, \quad \gamma_{a}=\frac{a_{\mathrm{max}}-a_{\min }}{2}, \quad\left|\Delta_{a}\right|<1
\end{gathered}
$$

We choose the output multiplicative form of uncertainty modelling to represent the relative error in the model

$$
G_{\mathrm{p}}(s)=\bar{G}(s)\left(1+\Delta_{\mathrm{m}}(s)\right)=\frac{b_{\text {nom }}}{s-a_{\text {nom }}}\left(1+\Delta_{\mathrm{m}}(s)\right)
$$

where

$$
\Delta_{\mathrm{m}}(s)=\frac{G_{\mathrm{p}}(s)-\bar{G}(s)}{\bar{G}(s)}=\frac{\Delta b s}{b_{\text {nom }}\left(s-a_{\text {nom }}-\Delta a\right)}
$$

The block diagram of the uncertainty model is represented in Figure 3. 


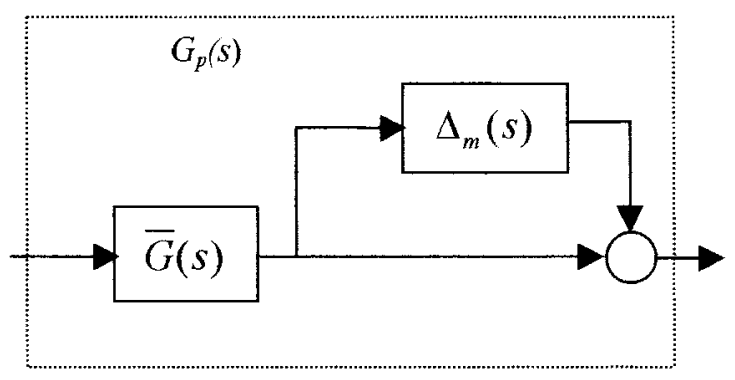

Figure 3. Block diagram of output multiplicative uncertainty

\section{PERFORMANCE SPECIFICATIONS}

A properly designed control system must track the time-varying reference input $X_{\text {Rref }}$ with small error and reject the external disturbance and noise inputs. In the present case $F_{\text {in }}$ is a measured disturbance and $S_{\text {in }}$ is an unmeasured disturbance. The output of the closed-loop system (Figure 2) is

$$
X_{\mathrm{R}}(s)=\frac{K(s) \bar{G}(s)}{1+K(s) \bar{G}(s) G_{\mathrm{m}}(s)} X_{\mathrm{Rref}}(s)+\frac{1}{1+K(s) \bar{G}(s) G_{\mathrm{m}}(s)} d(s)-\frac{K(s) \bar{G}(s)}{1+K(s) \bar{G}(s) G_{\mathrm{m}}(s)} n(s)
$$

Defining the tracking error as

$$
e(s)=X_{\mathrm{Rref}}(s)-X_{\mathrm{m}}(s)
$$

leads to

$$
e(s)=S(s) X_{\mathrm{Rref}}(s)-S(s) d(s)+T(s) n(s)
$$

where $S(s)=1 /\left(1+K(s) \bar{G}(s) G_{\mathrm{m}}(s)\right)$ is the sensitivity function, and $T(s)=K(s) \bar{G}(s)$ / $\left(1+K(s) \bar{G}(s) G_{\mathrm{m}}(s)\right)$ is the complementary sensitivity function.

Before all other specifications the control system has to guarantee stability despite model uncertainty. According to the small gain theorem ${ }^{24}$ if the complementary sensitivity function $T(s)$ and the uncertainty model $\Delta_{\mathrm{m}}(s)$ are stable the closed-loop system will be robustly stable if

$$
\left|\Delta_{\mathrm{m}}\right|<\frac{1}{|T|}, \quad\left|\Delta_{\mathrm{m}} T\right|<1
$$

From equation (19) it can be concluded that the sensitive function $S$ must be small in order to reduce the effect of disturbance and to keep the error small. The smaller the function values, the better the tracking. Therefore, tracking and disturbance rejection are compatible specifications. For proper suppression of noise we need to keep the complementary sensitivity function small. Because $S$ and $T$ must add to unity it means that noise immunity and the previous requirements are conflicting objectives. In a $H$-inf control framework the specifications just mentioned are defined using frequency-dependent weights covering different frequency ranges and thus avoiding the conflict. ${ }^{25}$ The requirement for the sensitivity function is formulated as specifying that $S(s)$ remains below a given frequency-dependent weight, i.e.

$$
|S| \leqslant W_{s}^{-1}, \quad \text { or } \quad\left|W_{s} S\right| \leqslant 1
$$




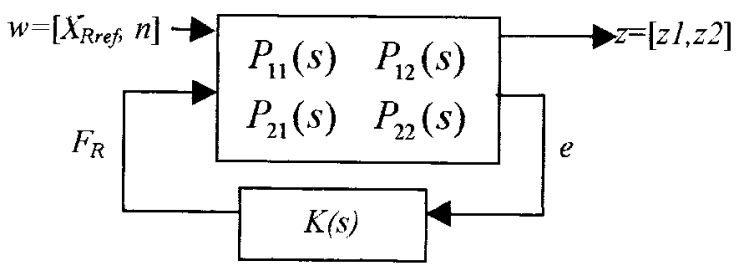

Figure 4. Linear Fractional Transformation (LFT) model

Similarly, $T(s)$ is specified to be kept below a given weight in the high-frequency range, i.e.

$$
|T| \leqslant W_{T}^{-1}, \quad \text { or }\left|W_{T} T\right| \leqslant 1
$$

Finally, both requirements can be satisfied by solving the so-called mixed sensitivity problem. Proper selection of the weights depends on the particular physics of the problem. The process in analysis is known to have a periodic behaviour with a period of about $24 \mathrm{~h}$. Consequently, a high weight can be placed near $\pi / 12\left(\mathrm{~h}^{-1}\right)$ to force $S(s)$ to a low value. The control system should be able to attenuate variations with a period larger than $2 \mathrm{~h}$. This corresponds to choosing a bandwidth of $\pi\left(\mathrm{h}^{-1}\right)$. The respective size of the largest anticipated perturbation is defined by $W_{T}(s)$. Therefore, we have to find first the bound of the multiplicative uncertainty, $\left|\Delta_{\mathrm{m}}\right| \leqslant w(s)$, and consequently $W_{T}=w(s)$.

The particular structure of $W_{T}(s)$ depends also on whether the original plant is only proper (i.e. has equal number of poles and zeros) or is strictly proper (more poles than zeros). In the latter case, which happens more frequently, we have to add to $W_{T}$ as many zeros as required so that $W_{T}(s) G(s)$ becomes a proper structure. That is why, usually, $W_{T}(s)$ is an improper transfer function (has more zeros than poles) and cannot be realized in state-space form, but $W_{T}(s) G(s)$ has state-space realization. An important consideration, when choosing the two weighting functions, is that the $0 \mathrm{~dB}$ crossover frequency of $W_{s}(\mathrm{j} \omega)$ Bode plot should be below the $0 \mathrm{~dB}$ crossover frequency of $W_{T}(\mathrm{j} \omega)$ Bode plot, otherwise the performance requirements will not be achieved.

\section{Two-port state-space representation of the augmented process model}

After weighting function selection the control system of Figure 2 has to be transformed in the two-port diagram shown in Figure 4. The original system is single-input single-output (SISO) whereas the new formulation will be a multi-input multi-output (MIMO) structure. The closed-loop transfer function between the regulated outputs $z$ and the exogenous inputs $w$ is

$$
z=\left\lfloor P_{11}+P_{12} K\left(I-P_{22} K\right)^{-1} P_{21}\right\rfloor w=T_{z w} w
$$

where $T_{z w}$ is called the Linear Fractional Transformation (LFT). The state-space description of LFT in packed-matrix form is finally given by

$$
P(s) \equiv\left[\begin{array}{ccc}
A & B_{1} & B_{2} \\
C_{1} & D_{11} & D_{12} \\
C_{2} & D_{21} & D_{22}
\end{array}\right]
$$

For the present case study the corresponding structure of the LFT model developed is presented in Section 6. 


\section{5. $H$-inf ROBUST CONTROL DESIGN}

The major difficulties in control system design can be attributed to the presence of uncertainty: uncertainty in the models used to represent the process and uncertainty in the disturbance and noise signals influencing the system. Robust control refers to the maintenance of designed specifications in the presence of uncertainty. The $H$-inf robust design approach is based on minimizing over frequency the peak values of certain system transfer functions, chosen by the engineer to represent the design objectives. ${ }^{26}$ The aim is to minimize the size (i.e. the $H_{\infty}$ norm) of a stable proper (matrix) transfer function (TF). In case of scalar TF, its $H_{\infty}$ norm is the maximum modulus of the frequency response over all real frequencies. If matrix TF is considered, then its $H_{\infty}$ norm is the maximum of the largest singular value over all real frequencies. Minimizing the $H_{\infty}$ norm is equivalent to minimizing the maximum possible energy in regulation error over the set of all possible disturbances. This physical interpretation of the $H_{\infty}$ norm, as maximum energy gain, is particularly useful in reducing the effect of uncertain disturbance signals. In fact, the disturbance is assumed to belong to a pre-specified set of signals and the energy of the output is to be minimized for the worst disturbance in the set, subject to the constraint of closed-loop stability. The $H_{\infty}$ norm is also significant in reducing the effects of model uncertainty. The unknown perturbation, representing parameter variations and/or unmodelled dynamics, is supposed to be bounded. By minimizing the $H_{\infty}$ norm, of the perturbed closed-loop TF for the worst case effect of uncertainty is possible to find the size of the smallest perturbation for which the system becomes unstable. The $H_{\infty}$ theory provides a reliable procedure for synthesizing a controller to optimize the performance and/or robust stability margins depending on the particular cost function used. $^{27,28}$ The weights in the cost function, in a general sense, have to shape over frequency the maximum singular values of the closed-loop $\mathrm{TF}$ of interest $(S, T)$. If there exist a feasible controller that meets the frequency domain constraints, a very precise loop shaping can be achieved. The procedure to solve the problem is carried out employing the two Riccati equation approach. ${ }^{24,29}$ Therefore, the $H_{\infty}$ control is considered as a frequency-domain design method that uses state-space machinery for computation. The goal is to find an internally stabilizing controller $K(s)$ for the two-port plant $P(s)$ such that the infinity norm of the closed-loop transfer function $T_{z w}(s)$ is below a given level $\gamma$ (a positive scalar) or find an internally stabilizing controller which minimizes the infinity norm of $T_{z w}(s)$. We start with the standard $H$-inf control problem to find a positive value for $\gamma$ and then apply an iterative procedure to reduce it until the problem fails to have a solution (optimal $H$-inf control). For the particular problem all assumptions concerning the existence of a solution are satisfied:

(a) The pair $\left(A, B_{2}\right)$ is stabilizable and $\left(C_{2}, A\right)$ is detectable. These assumptions are necessary for a stabilizing controller to exist.

(b) $\operatorname{rank} D_{12}=1$ (the same as the dimension of control input $u$ ) and $\operatorname{rank} D_{21}=1$ (the same as the dimension of controlled output $y$ ). These conditions are needed to ensure that the controller is proper.

(c) $\operatorname{rank}\left(\begin{array}{cc}A-\mathrm{j} \omega I & B_{2} \\ C_{1} & D_{12}\end{array}\right)=\operatorname{dim} x+\operatorname{dim} u=2$

(d) $\operatorname{rank}\left(\begin{array}{cc}A-\mathrm{j} \omega I & B_{1} \\ C_{2} & D_{21}\end{array}\right)=\operatorname{dim} x+\operatorname{dim} y=2$

(e) $D_{11}=0$ and $D_{22}=0$ 
Instead of writing the Riccati equations we will specify their associated Hamiltonian matrices, viz: The estimator Riccati equation Hamiltonian

$$
H_{\mathrm{e}}=\left[\begin{array}{cc}
A-B_{2} \tilde{D}_{12} D_{12}^{*} C_{1} & \gamma^{-2} B_{1} B_{1}^{*}-B_{2} \tilde{D}_{12} B_{2}^{*} \\
-\tilde{C}_{1}^{*} \tilde{C}_{1} & -\left(A-B_{2} \tilde{D}_{12} D_{12}^{*} C_{1}\right)^{*}
\end{array}\right]
$$

and the controller Riccati equation Hamiltonian

$$
H_{\mathrm{c}}=\left[\begin{array}{cc}
\left(A-B_{1} D_{21}^{*} \tilde{D}_{21} C_{2}\right)^{*} & \gamma^{-2} C_{1}^{*} C_{1}-C_{2}^{*} \tilde{D}_{21} C_{2} \\
-\widetilde{B}_{1} \tilde{B}_{1}^{*} & -A+B_{1} D_{21}^{*} \tilde{D}_{21} C_{2}
\end{array}\right]
$$

where

$$
\begin{array}{cl}
\tilde{D}_{12}=\left(D_{12}^{*} D_{12}\right)^{-1}, & \tilde{D}_{21}=\left(D_{21} D_{21}^{*}\right)^{-1} \\
\tilde{C}_{1}=\left(I-D_{12} \tilde{D}_{12} D_{12}^{*}\right) C_{1}, & \tilde{B}_{1}=B_{1}\left(I-D_{21}^{*} \tilde{D}_{21} D_{21}\right)
\end{array}
$$

The solutions of equations (25) and (26) are denoted by

$$
X_{\infty}=\operatorname{Ric}\left(H_{\mathrm{c}}\right) \text { and } Y_{\infty}=\operatorname{Ric}\left(H_{\mathrm{e}}\right)
$$

Necessary and sufficient conditions for a stabilizing controller to exist are that $X_{\infty}$ and $Y_{\infty}$ are positive-semi-definite solutions and the spectral radius $\rho\left(X_{\infty} Y_{\infty}\right)<\gamma^{2}$.

The control law is given by

$$
u=-K_{\mathrm{c}} \hat{x}
$$

with controller gain

$$
K_{\mathrm{c}}=\widetilde{D}_{12}\left(B_{2}^{*} X_{\infty}+D_{12}^{*} C_{1}\right)
$$

and the state estimator is

$$
\dot{\hat{x}}=\left(A+\gamma^{-2} B_{1} B_{1}^{*} X_{\infty}\right) \hat{x}+B_{2} u+Z_{\infty} K_{\mathrm{e}}\left(y-C_{2} \hat{x}-\gamma^{-2} D_{21} B_{1}^{*} X_{\infty} \hat{x}\right)
$$

with estimator gain

$$
K_{\gamma}=\left(Y_{\infty} C_{2}^{*}+B_{1} D_{21}^{*}\right) \tilde{D}_{21}
$$

Finally, the state-space description of the controller dynamics is given by

$$
\begin{aligned}
& A_{\mathrm{c}}=A-B_{2} K_{\mathrm{c}}-Z_{\infty} K_{\mathrm{e}} C_{2}+\gamma^{-2}\left(B_{1} B_{1}^{*}-Z_{\infty} K_{\mathrm{e}} D_{21} B_{1}^{*}\right) X_{\infty} \\
& B_{\mathrm{c}}=Z_{\infty} K_{\mathrm{e}}, \quad C_{\mathrm{c}}=-K_{\mathrm{c}}, \quad D_{\mathrm{c}}=0
\end{aligned}
$$

The design procedure can be summarized in the following way:

1. Write a two-port state-space formulation of the plant with the frequency weights.

2. Check if the assumption (the rank conditions) for existence of solution are satisfied. If they are not, go back to step 1 and reformulate the plant by changing the weights or adding inputs/outputs;

3. Select an initial positive value for $\gamma$;

4. Solve the controller and estimator Riccati equations;

5. Check if the solutions are positive semi-definite and the spectral radius condition is satisfied. If it is true go back to step 3 and reduce the value of $\gamma$, otherwise increase $\gamma$;

6. Stop the procedure if the optimal or acceptable solution is obtained. 
Table I. Constant numerical data

\begin{tabular}{lcccc}
\hline$V(1)$ & $T_{\mathrm{m}}(\mathrm{h})$ & $k_{\mathrm{m}}$ & $k_{\mathrm{ref}}\left(\mathrm{mgh} / 1^{2}\right)$ & $S_{\mathrm{in}}(\mathrm{mgCOD} / 1)$ \\
\hline $1.5 \times 10^{7}$ & $1 / 12$ & 1 & $3.8 \times 10^{-3}$ & 300 \\
\hline
\end{tabular}

Table II. Variable numerical data

\begin{tabular}{|c|c|c|c|c|}
\hline \multirow[b]{2}{*}{ Data } & \multirow{2}{*}{$\begin{array}{l}\text { Nominal } \\
\text { values }\end{array}$} & \multicolumn{2}{|c|}{ Variation bounds } & \multirow{2}{*}{$\begin{array}{l}\text { Sinusoidal variation } \\
\text { simulation }\end{array}$} \\
\hline & & Min & $\operatorname{Max}$ & \\
\hline$\mu_{\mathrm{m}}\left(\mathrm{h}^{-1}\right)$ & $0 \cdot 2$ & $0 \cdot 1$ & $0 \cdot 3$ & $\mu_{\mathrm{m}}=\mu_{\mathrm{m}}^{\mathrm{nom}}+0 \cdot 1 \sin (2 \pi t / 3+4 \pi / 3)$ \\
\hline$K_{\mathrm{m}}(\mathrm{mg} / 1)$ & 90 & 60 & 120 & $K_{\mathrm{m}}=K_{\mathrm{m}}^{\mathrm{nom}}+30 \sin (\pi t / 2)$ \\
\hline Y & $0 \cdot 6$ & $0 \cdot 5$ & $0 \cdot 7$ & $Y=Y^{\text {nom }}+0 \cdot 1 \sin (\pi t / 3+\pi / 3)$ \\
\hline$r$ & 4 & 3 & 5 & $r=r^{\mathrm{nom}}+\sin (\pi t / 6)$ \\
\hline$F_{\text {in }}(1 / \mathrm{h})$ & $1.9 \times 10^{6}$ & $0.75 F_{\text {in }}$ & $1.25 F_{\text {in }}$ & $F_{\text {in }}=F_{\text {in }}^{\text {nom }}(1+0.25 \sin \pi t / 12)$ \\
\hline$S_{\text {sub }}(\mathrm{mgCOD} / 1)$ & 2 & 1 & 4 & \\
\hline$X_{\mathrm{R}}(\mathrm{mg} / \mathrm{l})$ & 7070 & 4000 & 10000 & \\
\hline
\end{tabular}

\section{SIMULATION RESULTS}

The numerical data for the example are given in Tables I and II. The uncertainty of the kinetic parameters and the operating range were chosen to coincide with the typical range for domestic wastewater as reported by Sandstrom and Klei. ${ }^{1}$ After some iterations the actual weighting functions were chosen as

$$
W_{s}^{-1}=0 \cdot 01 \frac{200 s+1}{0 \cdot 2 s+1}, \quad W_{T}^{-1}=\frac{1}{s^{2}}
$$

The particular choice of the sensitivity weighting function corresponds to a steady-state tracking error of $1 \%$ because $W_{S}^{-1}(0)=0 \cdot 01$, and amplification of the high-frequency disturbances by a factor 10 , i.e. $W_{S}^{-1}(s \rightarrow \infty)=10$. The frequency range in which the sensitivity function is forced to be small is up to $\omega=0 \cdot 5[1 / \mathrm{h}]$, which includes the range of expected disturbances. Simultaneously, the choice of $W_{T}$ corresponds to a desired bandwidth of $2 \pi$ and forces the complementary sensitivity function to be small around this frequency where the measurement noise is amplified. The requirement for $0 \mathrm{~dB}$ crossover of the two weighting functions is also taken into account. Finally, the augmented two-port plant has the following structure:

$$
\left[\begin{array}{c}
z_{1} \\
z_{2} \\
e
\end{array}\right]=\left[\begin{array}{ccc}
W_{s} & -W_{s} G_{\mathrm{m}} & -W_{s} G_{\mathrm{m}} \bar{G} \\
0 & 0 & W_{T} \bar{G} \\
I & -G_{\mathrm{m}} & -G_{\mathrm{m}} \bar{G}
\end{array}\right]\left[\begin{array}{c}
X_{\mathrm{Rref}} \\
n \\
F_{\mathrm{R}}
\end{array}\right]
$$

where

$$
P_{11}=\left[\begin{array}{cc}
W_{s} & -W_{s} G_{\mathrm{m}} \\
0 & 0
\end{array}\right], \quad P_{12}=\left[\begin{array}{c}
-W_{s} G_{\mathrm{m}} \bar{G} \\
W_{T} \bar{G}
\end{array}\right], \quad P_{21}=\left[I-G_{\mathrm{m}}\right], \quad P_{22}=-G_{\mathrm{m}} \bar{G}
$$


By using MATLAB ${ }^{\mathrm{TM}}$ the above description is transformed in a state-space form with matrices

$$
\begin{aligned}
& A=\left[\begin{array}{ccc}
-12 \cdot 1150 & -1 \cdot 3800 & 0 \\
1 \cdot 0000 & 0 & 0 \\
0 & -0.0170 & -0 \cdot 0050
\end{array}\right], \quad B_{1}=\left[\begin{array}{l}
0 \\
0 \\
1
\end{array}\right], \quad B_{2}=\left[\begin{array}{l}
1 \\
0 \\
0
\end{array}\right] \\
& C_{1}=\left[\begin{array}{ccc}
0 & -0.0017 & 0.4995 \\
-0.2056 & -0.0234 & 0
\end{array}\right] \quad C_{2}=\left[\begin{array}{lll}
0 & -0.0170 & 0
\end{array}\right] \text {, } \\
& D_{11}=\left[\begin{array}{c}
0 \cdot 1000 \\
0
\end{array}\right], \quad D_{12}=\left[\begin{array}{c}
0 \\
0 \cdot 0170
\end{array}\right], \quad D_{21}=1, \quad D_{22}=0
\end{aligned}
$$

A numerical algorithm is implemented to find the optimal $H$-inf controller, starting with $\gamma=1$. The parameter $\gamma$ is the only parameter on which the iteration is performed. After several trials the minimum $\gamma$ was found to be $0 \cdot 79$. The following are the relevant data obtained:

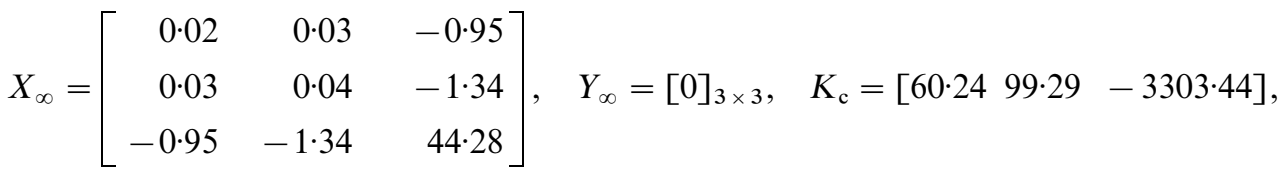

$$
\begin{aligned}
& K_{\mathrm{e}}=\left[\begin{array}{c}
0 \\
0 \\
1 \cdot 00
\end{array}\right]
\end{aligned}
$$

The final controller is stable and has the same number of states as the augmented plant with transfer function

$$
K(s)=\frac{3303 \cdot 44 s^{2}+4002 \cdot 14 s+4558 \cdot 74}{s^{3}+72 \cdot 36 s^{2}+101 \cdot 03 s+0 \cdot 5}
$$

The closed-loop poles $\{-12 \cdot 00,-0 \cdot 11,-0 \cdot 71+\mathrm{j} 0 \cdot 55,-0 \cdot 71-\mathrm{j} 0 \cdot 55,-70 \cdot 95\}$.

The results of the singular-value analysis are summarized in Figure 5. As shown, the cost function, $T_{z w}$, is all-pass (i.e. equal to $0 \mathrm{~dB}$, Figure 5(a)), the sensitivity function (Figure 5(b)) and the complementary sensitivity function (Figure 5(c)) approach their associated weighting functions, so that performance requirements (21) and (22) hold. The fixed controller not only stabilizes the feedback system but also maintains robust performance in the presence of all anticipated uncertainties and nonlinearities.

Tests of controller performance were carried out through simulation of the whole nonlinear system employing MATLAB/SIMULINK ${ }^{\mathrm{TM}}$. The numerical integration of the nonlinear equations (1)-(2) is based on the 5th-order Runge-Kutta method. In order to take into account the physical limitations for the real actuator saturation bound of $10^{6}\left[\mathrm{~h}^{-1}\right]$ is added in the simulations. The effect of model uncertainty is incorporated by imposing sinusoidal variations in the parameters and signals included in Table II. The amplitudes are selected so that the entire range of variation between known min and max values is covered. A measurement noise with variance 1200 is added in the simulations. 


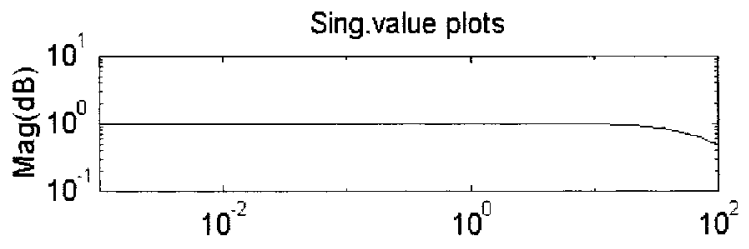

a

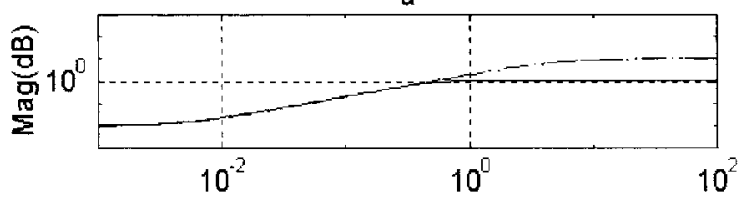

b

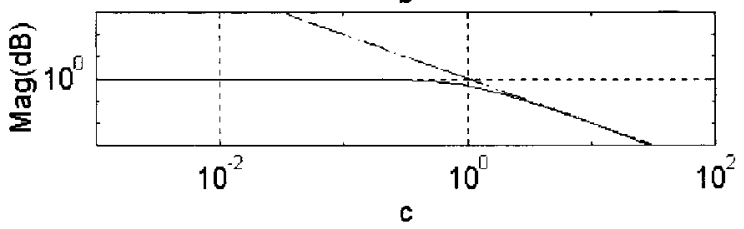

Figure 5. Frequency-domain characteristics: (a) $T_{z w}(\mathrm{j} \omega)$; (b) $S(\mathrm{j} \omega)$ and $W_{S}^{-1}(\mathrm{j} \omega)$ (dashed); (c) $T(\mathrm{j} \omega)$ and $W_{T}^{-1}(\mathrm{j} \omega)$ (dashed)
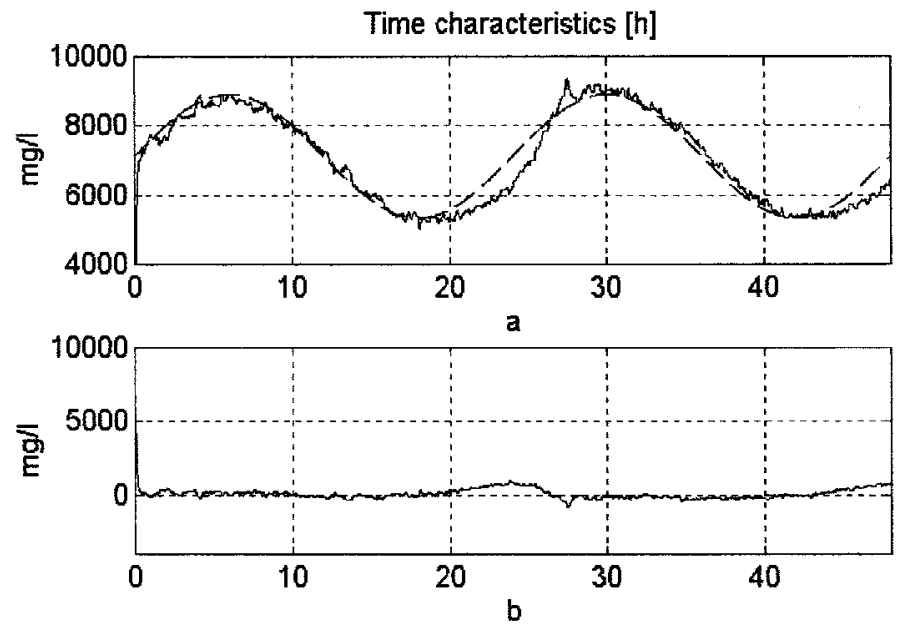

Figure 6. Tracking performance of the process with $H$-inf controller: (a) reference signal (dashed) and biomass concentration in the recycle flow; (b) tracking error

As shown in Figure 6 the biomass concentration of the recycle flow tracks the time-varying reference signal with a maximum error of $7 \%$. The time trajectories of the recycle flow rate and the substrate concentration are presented in Figure 7. The dynamic trajectory of organic solids stays within the operation window of $S_{\text {sub }}$ as specified in Table II.

For the purpose of comparison, process operation with a conventional fixed structure (PI) controller was equally simulated. The parameters of the PI controller were tuned by trial and 

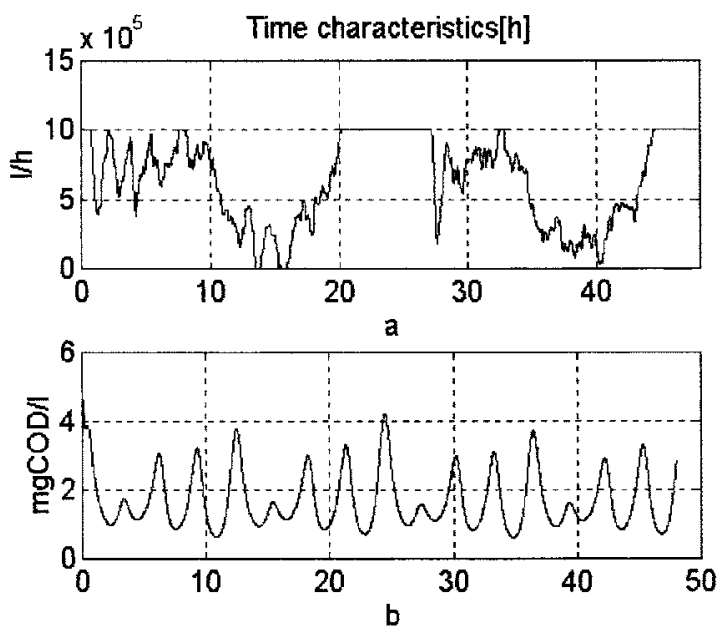

Figure 7. Control action and substrate trajectory with $H$-inf controller: (a) control signal; (b) substrate concentration
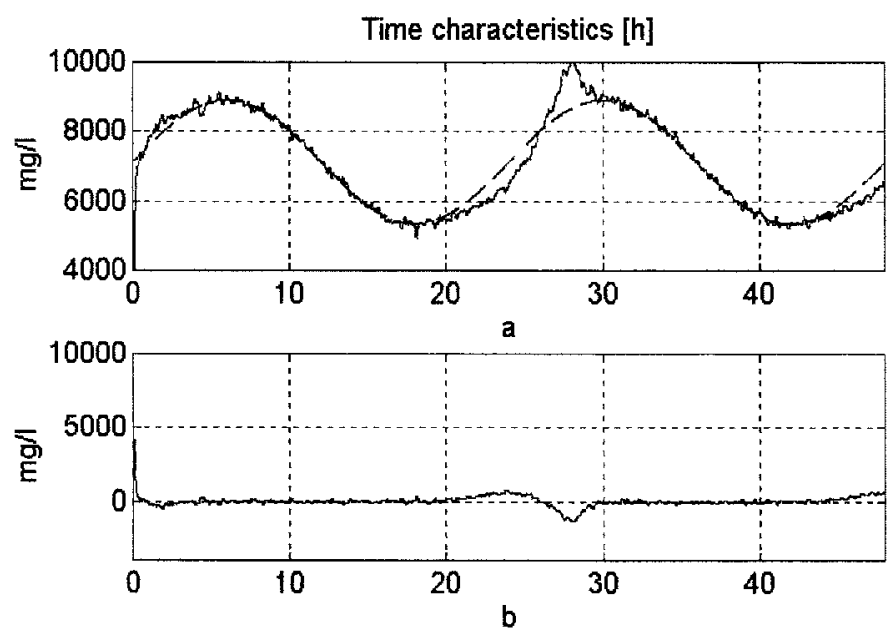

Figure 8. Tracking performance of the PI controlled process: (a) reference signal (dashed) and biomass concentration in the recycle flow; (b) tracking error

error with respect to the magnitude of the sensitivity and complementary sensitivity functions. The best possible tracking (Figure 8) was reached for PI controller with parameters $\left.k_{\mathrm{p}}=10^{4}(1 / \mathrm{h}) /(\mathrm{mg} / \mathrm{l})\right]$ and $T_{1}=0.83 \quad(\mathrm{~h})$ (assuming the controller equation is written as $\left.u(t)=k_{\mathrm{p}}\left(e(t)+1 / T_{1} \int e(t) \mathrm{d} t\right)\right)$. As expected, increasing the gain, to meet the tracking conditions, means larger bandwidth and consequently higher influence of the noise. This causes saturation problems, as shown in Figure 9. Furthermore, from a practical point of view, the control action is 

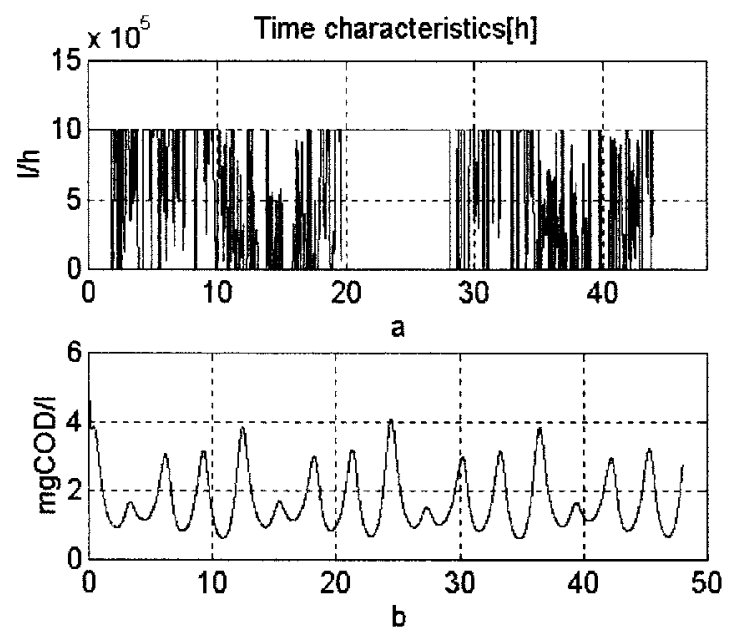

Figure 9. Control action and substrate trajectory of the PI controlled process: (a) control signal; (b) substrate concentration

also quite unsatisfactory since such high frequency actions are not appropriate in industrial operation.

\section{Remark}

In this case study we have chosen a two-port structure of the biological process in which the number of the outputs $z$ and the number of the exogenous inputs $w$ are equal (the so-called one-block structure). Very often this is not the case. For example, if we wish to prevent saturation problems in the actuator by penalising the control variable, then this variable should be added as an element of the output vector $z$ (Figure 4). The dimension of $z$ and $w$ would then not be the same and this would bring in computational problems. To avoid such difficulties the LFT structure should be reformulated by adding fictitious inputs or weights.

\section{CONCLUSIONS}

In this work we report an extension of the application areas of $H$-inf robust control theory to activated sludge processes. A robust controller was designed and tested with good results.

The $H$-inf control design was based on a simplified linear model together with the description of the nonlinearities by bounded uncertainty. The main problem of finding a reliable procedure to estimate nonmeasured variables was avoided since the algorithm adopted embeds an estimation of the states by solving the Riccati equation. The difficulty with parameter estimation was also avoided by assuming their variability within known bounds.

The controller performance was evaluated on its own and relatively to the observed behaviour of a conventional PI algorithm. 
The robust controller was able to force the system in tracking a time-varying processdependent reference signal for the concentration of substrate in the recycle stream. It performed well under restrictive conditions of measurement noise, load disturbances and actuator constraints.

The behaviour of the PI controller, in terms of tracking ability, was not significantly worst. However, its control action was seen not to be physically feasible. Moreover, considering the long time constants and random influent disturbances, systematic tuning of the conventional controller would be required.

One of the well-known problems relating to the application of $H$-inf robust control is the high order of the controller (which depends on the order of the selected weighting functions and on the order of the process itself). It is often advisable to apply a procedure of order reduction to obtain a final controller more suitable for practical implementation. Research work involving such a procedure for the particular problem of controlling an activated-sludge bioreactor is in progress and results will be reported in the near future.

\section{NOTATION}

$k_{\text {ref }} \quad$ reference proportionality factor

$k_{\mathrm{m}} \quad$ sensor parameter

$n \quad$ measurement noise

$r \quad$ output-input ratio of the settler

$F_{\text {in }} \quad$ influent flow rate $(1 / \mathrm{h})$

$F_{\mathrm{R}} \quad$ recycle flow rate $(1 / \mathrm{h})$

$\bar{G}(s) \quad$ process transfer function with nominal parameters

$G_{\mathrm{m}}(s) \quad$ sensor transfer function

$G_{\mathrm{p}}(s) \quad$ process transfer function

$K(s) \quad$ controller transfer function

$S_{\text {in }} \quad$ influent substrate concentration (COD mg/l)

$S_{\text {sub }} \quad$ substrate concentration (COD $\left.\mathrm{mg} / \mathrm{l}\right)$

$S(s) \quad$ sensitivity function

$T(s) \quad$ complementary sensitivity function

$T_{\mathrm{m}} \quad$ sensor time-constant

$Y \quad$ yield coefficient

$V \quad$ bioreactor volume (1)

$W_{s}(s) \quad$ performance weighting function

$W_{T}(s) \quad$ performance weighting function

$X \quad$ biomass concentration $(\mathrm{mg} / \mathrm{l})$

$X_{\mathrm{m}} \quad$ measured biomass concentration (mg/l)

$X_{\mathrm{R}} \quad$ concentration of the biomass in the recycle stream, $(\mathrm{mg} / \mathrm{l})$

$X_{\mathrm{Rref}} \quad$ reference signal for $X_{\mathrm{R}}(\mathrm{mg} / \mathrm{l})$

$\Delta_{\mathrm{m}}(s) \quad$ multiplicative uncertainty

$\mu \quad$ specific growth rate $(1 / \mathrm{h})$

\section{ACKNOWLEDGEMENTS}

This work was developed during the stay of the first author in Porto, at the Department of Chemical Engineering and at the Institute for Systems and Robotics, Faculty of Engineering, 
University of Porto. The author is deeply grateful for the hospitality of all the colleagues and the excellent working conditions at the host institutions. The stay was financially supported by a NATO Science Fellowship, Outreach Programme, contract No 2540, which is gratefully acknowledged. The research was also supported by the National Foundation "Scientific Investigations", Bulgarian Ministry of Education and Science, contract No. TN-715/97.

\section{REFERENCES}

1. Sundstrom, D. W. and H. E. Klei, Wastewater Treatment, Prentice-Hall, Englewood Cliffs, NJ, 1979.

2. Tchobanoglous, G. and F. Burton, Wastewater Engineering-Treatment, Disposal and Reuse, Metcalf \& Eddy, Inc, 3rd edn, McGraw-Hill Series in Water Resources and Environmental Engineering, NY, 1991.

3. Patry, G. G. and D. Chapman, Dynamic Modelling and Expert Systems in Wastewater Engineering, Lewis Publishers, Michigan, 1989.

4. Dold, P. L. and G. v. R. Marais, 'Evaluation of the general activated sludge model proposed by the IAWPRC task group', Water Sci. Technol., 18, 63-89 (1986).

5. Griffiths, P., 'Modification to the IAWPRC task group general activated sludge model', Water Res., 28(3), 657-664 (1994).

6. Arden, E. and W. T. Lockett, 'Experiments on the oxidation of sewage without the aid of filters', J. Soc. Chem. Ind., 33, 553, 1122 (1914).

7. Benefield, L. D. and C. W. Randall, 'Activated sludge and its process modifications', Biological Process Design for Wastewater Treatment, Prentice-Hall, Englewood Cliffs, NJ, 1980.

8. Marsili Libelli, S., 'Modelling, identification and control of the activated sludge process', Advances in Biochemical Engineering/Biotechnology, Springer-Verlag, Berlin, Heidelberg, Vol. 38, 1989, pp. 90-148.

9. Bastin, G. and D. Dochain, On Line Estimation and Adaptive Control of Bioreactors, Elsevier Science Publishers, Amsterdam, 1990.

10. Aitken, M. D., Ph. E. Heck, L. Alvarez-Cohen, S. J. Grimberg and W. T. Stringfellow, 'Activated sludge', Water Environ. Res., 65(4) 324-336 (1993).

11. Andrews, J. F. et al., Dynamics and Control of the Activated Sludge Process, Water quality management library, Vol. 6 , Technomic Publishing, 1992.

12. Schaper, C., D. Mellichamp and D. Seborg, 'Robust control of a wastewater treatment system', Proc. 29th Conf. on Decision and Control, December, Honolulu, Hawaii, 1990.

13. Olsson, G., B. Andersson, B. G. Hellstrom, H. Holmstrom, L. G. Reinuis and P. Vopatek, 'Measurements, data analysis and control methods in wastewater treatment plant - state of the art and future trends', Water Sci. Tech., 21, 1333-1345 (1989).

14. Ferreira, E. C. and S. Feyo de Azevedo, 'Adaptive linearising control of bioreactors', Proc. CONTROL '96-UKACC Int. Conf. on Control, 1184-1189, Exeter, U.K., 2-5 September, 1996.

15. Christen, U., H. E. Musch and A. Henson, 'Robust control of distillation columns: $\mu$-vs $H_{\infty}$-synthesis', J. Process Control, 1, 19-30 (1997).

16. Murad, G., I. Postlethwaite and Da-Wei Gu, 'A discrete-time internal model based $H_{\infty}$ controller and its application to a binary distillation column', J. Process Control, 6, 451-465 (1997).

17. Steffens, M. A., P. A. Lant, R. B. Newell and I. T. Cameron, 'Dynamic modelling of activated sludge processes: a new modular simulator', Proc. PSE'94, 1994, pp. 323-326.

18. Lessard, P. and M. B. Beck, 'Dynamic modelling of the activated sludge process: a case study', Water Res., 27(6), 963-978 (1993).

19. Verstraete, W., E. van Vaerenbergh, Aerobic Activated Sludge, ch. 2 in Biotechnology, Vol. 8: Microbial Degradations W. Schoborn (Vol. Ed.), VCH, Weinheim, 1986, pp. 43-112.

20. Kokotovic, P., H. K. Khalil and J. O'Reilly, Singular Perturbations Methods in Control: Analysis and Design, Academic Press, London, 1986.

21. Oliveira, R., E. Ferreira, F. Oliveira and S. Feyo de Azevedo, 'A Study on the convergence of observer-based kinetics estimators in stirred tank bioreactors', J. Process Control, 6(6), 367-371 (1996).

22. Pelkonen, M. and R. Tenno, 'New control parameters and measurement techniques for the activated sludge process', Water Sci. Tech., 27(5-6), 287-295 (1993).

23. Tenno, R., R. Vilu and H. Oit, 'Stochastic control for stabilisation of sludge loading characteristics in an aerobic wastewater treatment system', Automatica, 25, 739-742 (1989).

24. Doyle, J. C., K. Glover, P. Khargonekar and B. Francis, 'State-space solution to standard H2 and $H$-inf control problems', IEEE Trans. Automat. Control, AC-34, 832-847 (1989).

25. Georgieva, P. G. and M. N. Ignatova, LFT models of continuous biotechnological process. J. Bioprocess Engng., 20(2), 179-183 (1999) 
26. Postlethwaite, I., 'Introduction to H-inf design', Advanced Control Course, Ch. 10, Cambridge Control Limited, 1992.

27. Georgieva, P. G. and M. N. Ignatova, ' $H$-inf robust control of continuous biotechnological process', 2nd Eur. Symp. on Biochemical Engineering Science, Porto 16-19 September, Portugal, 1998.

28. Georgieva, P., Robust multivariable system analysis and design in the frequency domain, Ph.D. Thesis, Publishing House of Technical University-Sofia, 1997.

29. Khargonecar, P., I. R. Petersen and M. A. Rotea, 'H-inf optimal control with state feedback', IEEE Trans. Automat. Control, AC-33, 783-786 (1988). 